\title{
Survival and Success of Autotransplantation Maxillary Canine with Closed Apex: Systematic Review
}

\author{
Lamia Bouchghel, Kenza Khamlich, Farid Bourzgui, Farid El Quars \\ Orthodontics Department, Faculty of Dentistry, University Hassan II, Casablanca, Morocco \\ Email: lamiabouchghel@gmail.com
}

How to cite this paper: Bouchghel, L., Khamlich, K., Bourzgui, F. and El Quars, F. (2022) Survival and Success of Autotransplantation Maxillary Canine with Closed Apex: Systematic Review. Open Access Library Journal, 9: e8417.

https://doi.org/10.4236/oalib.1108417

Received: February 2, 2022

Accepted: February 21, 2022

Published: February 24, 2022

Copyright $\odot 2022$ by author(s) and Open Access Library Inc.

This work is licensed under the Creative Commons Attribution International License (CC BY 4.0).

http://creativecommons.org/licenses/by/4.0/

(c) (i) Open Access

\begin{abstract}
The frequency of canine retention varies between $0.9 \%$ and $2.2 \%$. When surgical exposure and subsequent orthodontic realignment are difficult or impossible due to unfavorable impaction position of an impacted maxillary canine or when the patient refuses prolonged orthodontic treatment, autotransplantation becomes a valuable alternative. The aim of this systematic review was to determine the level of success of maxillary canine autotransplantation, long-term stability, and factors involved in the outcome of this therapy. A literature search for all data published with 10 years limitation and no restriction of language was conducted, using: PubMed, Scopus, Web of Science, Medline Complete (EBSCO) and Cochrane. Specific journals in the area were also consulted Inclusion and exclusion criteria were specified. A total of 5 studies were included in the present analysis. Of the 5 studies, 3 were retrospective and 2 were prospective. For outcomes, the results with reference to teeth, alveolar bone, periodontal tissues, and esthetic satisfaction were considered. The mean age of the participants ranged from 13 to 42.1 years old. Risk of bias was evaluated using the methodological index for nonrandomized studies-MINORS. The studies with a follow-up of more than 5 years presented a survival rate higher than $83 \%$ and the success rate was: $38 \%$. Studies with a follow-up of less than 5 years, survival rate was closer to $100 \%$ for, with success rate higher than $68 \%$. The overall success rate and survival were high, despite the methodologic limitations of the included studies. There is sufficient clinical experience to justify transalveolar transplantation of maxillary canines, with closed apices, as a legitimate treatment alternative considering the proper indication. Further study is needed of the prognostic factors that influence the success of autotransplantation with closed apex.
\end{abstract}

\section{Subject Areas}

Dentistry 


\section{Keywords}

Autotransplantation, Success Rate, Survival Rate, Orthodontic, Closed Apex, Maxillary Canine

\section{Introduction}

Children and young adults may often exhibit congenitally missing teeth or early loss of teeth due to trauma or caries [1]. The canine is the longest tooth in the oral cavity, and it is important for both esthetics and function. Its location at the curve of the dental arch is strategic for supporting the corner of the mouth, maintaining the arc smile, and protecting the periodontium within the lateral segments [2].

The incidence of canine impaction ranked second compared with molars. Furthermore, this impaction is more prevalent in women than in men with a 2:1 ratio. The frequency of canine retention varies between $0.9 \%$ and $2.2 \%$ [3]. The traditional treatment options for impacted canines are interceptive removal of the deciduous canine, surgical exposure with or without orthodontic traction to align the malpositioned tooth, no treatment, autotransplantation of the permanent canine or removal of the permanent canine and prosthetic or restorative treatment [2]. When surgical exposure and subsequent orthodontic realignment are difficult or impossible due to unfavorable impaction position of an impacted maxillary canine or when the patient refuses prolonged orthodontic treatment, autotransplantation becomes a valuable alternative [2].

Tooth autotransplantation is defined as the transplantation of an unerupted or erupted tooth in the same individual, from one site to another extraction site or a new surgically prepared socket [3]. Autotransplanted teeth have a vital periodontium and are compatible with alveolar growth [4] [5]. Immature teeth with open apices usually have sufficient blood supply as well as stem cells to promote pulp revascularization post-transplantation [6]. Pulp revascularization may allow continuous root development and maintenance of pulp vitality [7]. In addition, autotransplantation helps to maintain the natural shape of attached gingiva, which may result in a good aesthetic outcome [8]. Moreover, this technique is contraindicated in patients with cardiac anomalies, poor oral hygiene, lack of self-motivation and insufficient alveolar bone [3].

This procedure has become topical as it has been shown to provide functional adaptation, alveolar bone induction, preservation of the alveolar bone ridge and restauration of a normal alveolar process [2]. Nevertheless, dental transplantation is often perceived as an unpredictable alternative by its supporters. Thus, to try to verify the degree of accuracy of this assumption, the aim of this study was to perform a systematic review of the scientific literature regarding autotransplantation of permanent maxillary canines with emphasis on the level of success of this technique, long term stability and factors involved in the outcome of this therapy. 


\section{Materials and Methods}

\subsection{Focus Question}

This systematic analysis was realized to answer the focus question: What are the factors involved in the long term success and stability of the autotransplantation of permanent maxillary canines?

\subsection{Search Strategy}

The methodology applied in this systematic analysis was based on the PRISMA guidelines (Preferred Reporting Items for Systematic Review and Meta-Analysis).

A search protocol was specified in advance and registered at PROSPERO (International Prospective Register of Systematic Reviews) ID: CRD42021251765

To identify the relevant articles with 10 years limitation and no restriction of language, published up to December, 2021, a search was conducted in the following electronic databases: PubMed, Scopus, Web of Science, Medline Complete (EBSCO) and Cochrane. Specific journals in the area were also consulted. The MeSH descriptors/terms used are shown in Table 1.

As well as:

- Cross-references

- Grey literature:

Open Grey (http://www.opengrey.eu/): System for Information System for Grey Literature in Europe.

New York Academy of Medicine Grey Literature Report

(http://www.greylit.org/ between 1999-2016).

Clinical Trials.gov (US clinical trials registry).

\subsection{Eligibility Criteria of the Articles}

The eligibility criteria were determined in accords with the scheme of the patient/intervention/comparison/outcome study (PICO) question, inclusion, and exclusion criteria (Table 2).

\subsection{Data Collection and Analysis}

\subsubsection{Selection of Studies}

Titles and abstracts of relevant studies identified through the electronic searches

Table 1. Database and search method.

\begin{tabular}{ll}
\hline Database & Search strategy \\
\hline PubMed Scopus & ((orthodontics [MeSH Terms] OR orthodontic OR orthodontic \\
Web of Science & patients OR orthodontic treatment OR tooth movement OR teeth \\
Medline & movement) AND (transplantation [MeSH Terms] OR tooth \\
Complete & autotransplantation OR teeth autotransplantation OR autogenous \\
(EBSCO) & tooth transplantation OR autogenous teeth transplantation OR tooth \\
Cochrane & transplantation OR teeth transplantation) AND ((survival rate [MeSH \\
& Terms] OR success rate [MeSH Terms] OR efficacy OR side effects \\
& [MeSH Terms] OR collateral effects)) \\
\hline
\end{tabular}


Table 2. PICO criteria for study selection.

\begin{tabular}{ll}
\hline Participants (P) & $\begin{array}{l}\text { Orthodontic patient of any gender or malocclusion in the permanent } \\
\text { dentition with full development of the maxillary canine root. }\end{array}$ \\
Intervention (I) & $\begin{array}{l}\text { Autotransplantation of permanent maxillary canines } \\
\text { Comparison (C) }\end{array} \quad \begin{array}{l}\text { Non autotransplanted canine } \\
\text { Outcomes/Result (O) }\end{array}$
\end{tabular}

were screened by three authors (FB, LB, and $\mathrm{KK})$. Full-text articles were obtained from the studies that fulfilled the inclusion criteria. These full-text articles, together with full-text articles found through the manual search, were independently assessed by these authors to determine if they were in line with the inclusion criteria. Disagreements were resolved through discussion. After selection, data extraction and a risk-of-bias assessment were performed.

\subsubsection{Inclusion and Exclusion Criteria of Eligible Studies}

\section{1) Main inclusion criteria}

- Studies investigating the indication, success/survival rate, and surgical procedure of autologous transplantation of maxillary impacted canines.

- Controlled trials or prospective/retrospective studies.

- Studies reporting at least one of the following: survival rate (short or longterm), success rate, pulp condition, tooth mobility, presence of ankylosis, and root resorption of autotransplanted teeth with complete root formation.

- Mean follow-up period should be at least 2 years.

- No restrictions on language were made. 2) Main exclusion criteria

- Studies including autologous transplantation other than maxillary canines.

- Case reports, opinion articles, and review articles.

- Studies reporting autotransplantated teeth in patients with systemic diseases, syndromes, or cleft lip and palate.

- Animal studies.

- Studies without specified transplantation protocol.

- Studies referring to transplantation of traumatized maxillary canines.

- Studies reporting autotransplantation of teeth with a history of cysts, tumours, or trauma.

- Studies including sterilized teeth, cryopreserved teeth, or teeth maintained in culture media.

- Studies with patients that have genetic or systematic diseases.

- Studies not including information about follow-up or the success rate.

\subsubsection{Data Extraction and Management}

Data from the selected articles were independently extracted by the three reviewers (FB, $\mathrm{LB}$ and $\mathrm{KK}$ ); for this purpose, they used a personalized version of the Cochrane data extraction model for reviews [9]. After data collection, the information obtained from each study was organized in tables that examined the 
PICOS criteria. Methodological quality of eligible articles was assessed using the MINORS (Methodological index for non-randomized studies) based on the following scores: 0 - when unreported item, 1-when reported inappropriately, and 2-when reported properly.

The articles were classified based on their methodological quality: low ( $>17)$, medium $(\geq 10 \leq 17)$, and high risk of bias $(<10)$ (Table 3$)$.

\subsubsection{Risk of Bias in Included Studies}

On the basis of the MINORS quality assessment, all studies had a medium risk of bias (Table 3).

Table 3. Score of each article selected with the inclusion criteria according to the items of MINORS.

\begin{tabular}{|c|c|c|c|c|c|}
\hline MINORS score & $\begin{array}{l}\text { Patel et al. } \\
\quad(2011)\end{array}$ & $\begin{array}{c}\text { Grisar et al. } \\
\quad(2018)\end{array}$ & $\begin{array}{c}\text { A.B Hewitt et al. } \\
(2011)\end{array}$ & $\begin{array}{l}\text { Grisar et al. } \\
\quad(2020)\end{array}$ & $\begin{array}{c}\text { Huth et al. } \\
\quad(2013)\end{array}$ \\
\hline A clearly stated aim & 2 & 2 & 2 & 2 & 2 \\
\hline Inclusion of consecutive patients & 2 & 2 & 2 & 2 & 2 \\
\hline Prospective data collection & 0 & 0 & 0 & 2 & 0 \\
\hline End points appropriate to the aim of the study & 1 & 1 & 1 & 2 & 1 \\
\hline Unbiased assessment of the study end point & 1 & 1 & 1 & 1 & 1 \\
\hline Follow-up period appropriate to the aim of the study & 2 & 2 & 2 & 1 & 1 \\
\hline Loss of follow-up lower than $5 \%$ & 1 & 1 & 1 & 2 & 2 \\
\hline Prospective calculation of the study size & 1 & 1 & 2 & 1 & 1 \\
\hline Adequate control group & 2 & 2 & 2 & 2 & 2 \\
\hline Contemporary groups & 0 & 0 & 0 & 0 & 0 \\
\hline Baseline equivalence of groups & 0 & 0 & 0 & 0 & 0 \\
\hline Adequate statistical analyses & 2 & 2 & 2 & 2 & 2 \\
\hline Total score & 14 & 14 & 15 & 17 & 14 \\
\hline Risk of bias & Medium & Medium & Medium & Medium & Medium \\
\hline
\end{tabular}

One item received score: 0 -when not reported, 1 -when inadequately reported, and 2-when adequately reported. The articles were classified in accordance with their methodological quality into low $(>17)$, medium $(\geq 10 \leq 17)$, and high risk of bias $(<10)$.

\section{Results}

After screening titles and abstracts of 483 unique papers, 33 potentially eligible articles were selected (Figure 1: Flow diagram). Each title and abstract were independently reviewed by three researchers (FB, LB and $\mathrm{KK}$ ), and the obtained information was compared. Inter-examiner disagreements were resolved in a consensus meeting. Of the 33 potentially eligible articles, 28 were excluded. These articles were excluded for the following reasons: study with histological analysis; studies concerning auto-transplantation without specific results or conclusions about maxillary canines in a specific way; short paper about general reasons, requirements, treatment plans, and techniques of autotransplantation of 
Records identified through database searching 483 articles
Records identified through manual search 11 articles

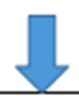

Records after duplicates removed $240+11$

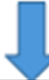

Records screened 251

Records excluded 197

Full text articles assessed for eligibility $\mathbf{5 4}$ articles

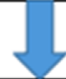

Full text articles excluded 49 articles

Based on the following reasons:

- Studies including autologous transplantation other than maxillary canines

- Case reports, opinion articles, and review articles

- Studies reporting autotransplanted teeth in patients with systemic diseases, syndromes, or cleft lip and palate

- Animal studies

- Studies without specified transplantation protocol

- Studies referring to transplantation of traumatized maxillary canines

- Studies reporting autotransplantation of teeth with a history of cysts, tumours, or trauma

- Studies including sterilized teeth, cryopreserved teeth, or teeth maintained in culture media

- Studies with patients that have genetic or systematic diseases

- Studies not including information about follow-up or the success rate

Figure 1. Flow diagram.

\section{Articles included for systematic review and meta-analysis : 5 articles}

maxillary canines without clinical outcomes; studies focusing on root anomalies of impacted maxillary canines; literature reviews; studies without information about survival and/or success rates.

\subsection{Included Studies}

A total of 5 articles were identified for inclusion in this review [10] [11] [12] [13] [14]. This systematic review was based on prospective and retrospective crosssectional studies due to the absence of controlled trials. The reported final outcomes, the survival and success rates from individual studies are summarized in Table 4.

\subsection{The Study Settings and Investigators}

Of the 5 included studies, two were performed in the UK [10] [12], two in Belgium [11] [13] and one was performed in Germany [14]. The studies were retrospective and prospective. 


\subsection{Participant Characteristics}

The mean age of the participants ranged from 13 to 42.1 years old (minimum age 9 and maximum age 51). One study did not report mean age [11]. The distribution of men and women was presented in 2 of the 5 studies. Between 17 and 235 patients were selected for each study. All the studies mentioned the number of teeth as well as the number of patients. Seventeen to 254 maxillary canines were selected for each study. Three studies solely reported on the transplantation of maxillary canines [10] [11] [13].

\subsection{Intervention Details}

\subsubsection{Pre-Operative Assessment}

Most of the studies did not report any details about clinical and radiographical pre-operative assessment. Root development stage was mentioned in all studies and found to be complete. Canine angulation and root anomalies were not mentioned in any studies. These studies mentioned severe impaction and difficult malpositioning, as such that orthodontic treatment was either impossible or would have been complicated and time-consuming.

\subsubsection{Operative Protocol}

The surgical procedures of maxillary canine transplantation were identical or similar to the protocol outlined by Andreasen et al. [15]. First, the surgical sites are disinfected and a local anaesthetic is injected. The remaining primary canine, if still present, is extracted, and a trapezoidal flap incision is made ensuring intact mesial, distal, and palatal gingiva at the graft site. To prepare the recipient socket, an osteotomy is performed using a surgical bur with water cooling. The socket for the graft should be slightly larger than the graft. Next, the crown of the impacted canine is exposed and the tooth removed with a periosteal elevator. The donor tooth is extracted slow and as atraumatically as possible. Next, the donor tooth is placed into the recipient socket without any pressure. The trapezoidal flap is repositioned and sutured.

Two studies mentioned the extra-oral time as an important stability factor [12] [13]. The fixation method was discussed in 2 studies [10] [12]. Occlusal positioning of the transplanted maxillary canine was discussed in 4 studies [10] [12] [13] [14] and found to be infra-occlusal.

\subsubsection{Post-Operative Assessment}

Most of the studies did not report any details about a clear healing protocol or the post-operative assessment other than including clinical and radiographic examinations. The duration and recurrence of clinical and radiological follow-up were mentioned in one study [12]. Two studies [11] [13] mentioned the (MCAI: Maxillary Canine Aesthetic Index) as an aesthetic evaluation index involved in the evaluation of the success rate. The AMCRI (Autotransplanted Maxillary Canine Radiological Index) was mentioned equally in these two studies.

Endodontic treatment and indications were discussed in all studies. In one 
study, [10] endodontic treatment was only indicated in case of signs of periapical infection or inflammatory root resorption.

\subsection{Outcome Measures}

As such, authors of this review preferred to to shed light on nuance existing between the survival rate and the success rate. The survival rate which was defined as the percentage of the autotransplanted maxillary canine present during the observation period. While the successful outcome was defined as the percentage of transplanted teeth still present and functioning well at the time of recall.

Bone-related outcomes were reported in all studies. Soft tissue outcomes were frequently tested by all studies in the clinical follow-up. Only one study [11] mentioned the deviation of the buccolingual inclination compared to the contralateral canine as an outcome to determine during the evaluation. Aesthetic outcome was reported in two studies [11] [13] by the MCAI index as an objective criterion to score the aesthetic outcome. Only one study [14] uses a questionnaire with numeric analog scales $(1=$ excellent; $6=$ unsatisfactory) to score patient satisfaction.

\subsection{Mean Duration of Follow-Up}

The mean duration of follow-up was found in all studies. In two studies, [13] [14] only a minimal follow-up time was mentioned. The mean time varied between 28 months and 26.5 years.

\subsection{Impact of Intervention}

Five studies presented outcome data for 455 autotransplanted maxillary canines (Table 4).

\section{Discussion}

The purpose of this study was to review transalveolar autotransplantation of maxillary canines and its long-term results. The investigated outcome parameters included survival rate, success rate and stability factor of dental auto transplantation. Prospective and retrospective studies identified accordance to strict inclusion criteria were included. Available information on indication and follow-up was

Table 4. Data extraction table for selected articles.

\begin{tabular}{|c|c|c|c|c|c|c|c|c|c|}
\hline Mean FU & Study & Survival rate & Success rate & Study design & $\mathrm{N}$ pt & $\mathrm{N}$ teeth & $\mathbf{M}$ & $\mathbf{F}$ & Mean age \\
\hline \multirow{3}{*}{$>5$ years } & Patel et al. [10] & $83 \%$ & $38 \%$ & Retrospective study & 49 & 63 & - & - & 21.8 years \\
\hline & Grisar et al. [16] & $67 \%$ & - & Retrospective study & 71 & 84 & - & - & - \\
\hline & A.B Hewitt et al. [12] & $97 \%$ after 10 years & - & Retrospective study & 235 & 254 & - & - & 15.4 \\
\hline \multirow{2}{*}{$<5$ years } & Grisar et al. [13] & $100 \%$ & $68 \%$ & Prospective study & 17 & 17 & 10 & 7 & 28 months \\
\hline & Huth et al. [14] & $96 \%$ & $74 \%$ & Prospective study & 45 & 37 & 26 & 19 & 1.6 years \\
\hline
\end{tabular}

M: Male. F: Female. $\mathrm{N}$ teeth: Number of teeth. $\mathrm{N}$ pt: Number of point. 
illustrated. 5 studies were included in this review with a moderate level of bias, most of these studies were retrospective (4 of 5). Also, it was evident that the literature lacks sufficiently well-organized studies concerning the topic of maxillary canines autotransplantation. There was a striking absence of clear-reported diagnostic pathways, pre-operative planning, and post-operative follow-up.

All studies included reported survival rates that were higher than $83 \%$ for studies with a follow-up of more than 5 years [10-12] and closer to $100 \%$ for studies with a follow-up of less than 5 years [13] [14]. None of the included studies discussed orthodontic movement after surgery. Concerning the surgical technique, there was more uniformity between the different studies. None of the studies explicitly reported careful handling of the follicle nor 3D planning.

The authors of this studies, agree that the criteria for success of an autotransplanted tooth consists of the absence of clinical and radiological pathology: absence of dental mobility or mobility lower than grade II, absence of pocket greater than 3 or $3.5 \mathrm{~mm}$, absence of inflammation, presence of pulpal vitality, good bone regeneration and absence of root resorption. Some authors [10] [11] [13] added the aesthetic factors as an important element of success (normal colour, no tooth recession or major deviation of the buccolingual inclination compared to the contralateral canine, good-to-excellent outcome compared to the contralateral biological erupted canine (MCAI/AMCRI)). Grisar et al. [11] [13] have used, in addition to aesthetic outcome MCAI, radiological index AMCRI, one of these studies [14] reports that patient satisfaction is also an important element and an effective way to evaluate the success of an autotransplantation. As there is lack of consensus regarding a set of universally accepted outcome criteria, studies of transalveolar transplanted canines should make an effort to describe parameters of clinical outcome. The criteria used for the assessment of final outcome in autotransplantation were quite variable ranging from the tooth simply being present intra-orally to present and completely free from resorption, discolouration, and pocketing, while maintaining vitality

Regarding the stability factor, the majority of studies (4/5) [10] [12] [13] [14] reported that postoperative occlusion was a very important stability factor. The atraumatic surgical technique was cited by two studies [10] [11], one of which was surgeon dependent. Two of the studies sought to minimize the extraoral time of the autograft. The authors [13] [14] mainly emphasized hygiene as a factor of stability, one study [10] prescribed antibiotics postoperatively and considered endodontic treatment and root maturation as a factor of stability, another study [14] considered patient's age and cigarette smoking.

All included studies report the use of clinical and radiological investigation as a mean of measurement, two of these studies [11] [13] added the aesthetic factor in the assessment. The studies reported the use of $2 \mathrm{D}$ radiographs for the assessment of root status, apex and canine position. However, no study mentioned the state of root angulation and any root anomalies. The use of 3D radiographic analysis on CBCT has not been reported in any study. Nowadays, there is a trend 
towards the use of 3D planning for autotransplantation, which allows precise positional planning with the possible use of computer assisted surgical guidance, greater ease of surgery and a reduction in extra-oral time. However, the quality of the existing evidence is low [16]. Previous studies have proven that regular follow-up with clinical check up and intraoral radiographs were sufficient in clinical practice [17]. However, in a research setting it might be interesting to use 3D imaging to evaluate tooth-related and bone-related outcomes in the short and long term.

Most of the studies [11] [12] [13] [14] only mentioned the duration and recurrence of clinical follow-up and the usage of 2D radiographs [10] [11] [12] [13] [14]. Most authors consider endodontic treatment of autotransplanted canines with closed apices as mandatory analogue of traumatically avulsed teeth with closed apices. However, other authors [18] [19] [20] also suggest a wait-and-see strategy even in cases of closed apices.

Better survival and success rated have been reported with autotransplanted teeth with open apex versus closed apex [21]. However, Chung et al. [18], in their systematic review of transplanted teeth with a closed apex, found high survival rates of 98 per cent at the 1-year follow-up point and 90.5 per cent at the 5-year follow-up point.

\section{Conclusions}

\subsection{Implications for Practice}

Based on the findings of the current study, it became clear that the literature is deficient in high-quality clinical studies. There is sufficient clinical experience to justify transalveolar transplantation of maxillary canines, with closed apices, as a legitimate treatment alternative considering the proper indication.

More standardization is needed for clinical assessment parameters, clinical benefits, and risks of orthodontic movement of auto-transplanted teeth, and an approach to aesthetic satisfaction as well as quality of life of patients undergoing this treatment.

\subsection{Implications for Research}

Randomized Controlled Trials (RCTs) with sample size calculations are recommendable to support more plausible conclusions. However, due to the specifies of autotransplantation, in addition to factors such as age, patient expectations, prosthetic rehabilitation options and orthodontic treatment planning, it may be difficult to conduct RCTs. Therefore, future high-quality longitudinal observational studies may provide meaningful results.

\section{Declarations}

\section{Ethics Approval and Consent to Participate}

Approval for conducting the study was obtained from the Research Ethics Committee of Casablanca College of Dentistry. 


\section{Availability of Supporting Data}

The datasets used and/or analysed during the current study are available from the corresponding author.

\section{Authors' Contributions}

LB: Substantially contributed to conception or design, to acquisition, analysis, or interpretation of data/Agree to be accountable for all aspects of the work in ensuring that questions relating to the accuracy or integrity of any part of the work are appropriately investigated and resolved/Prepared and critically revised the manuscript/Corresponding author.

KK: Substantially contributed to conception or design, to acquisition, analysis, or interpretation of data/Agree to be accountable for all aspects of the work in ensuring integrity and accuracy/Gave final approval.

FB: Substantially contributed to conception or design, to acquisition, analysis, or interpretation of data/Critically revised the manuscript/Gave final approval.

FE: Contributed to conception or design, to acquisition, analysis, or interpretation of data.

\section{Conflicts of Interest}

The authors declare no conflicts of interest.

\section{References}

[1] Machado, L.A., do Nascimento, R.R., Ferreira, D.M., Mattos, C.T. and Vilella, O.V. (2016) Long-Term Prognosis of Tooth Autotransplantation: A Systematic Review and Meta-Analysis. International Journal of Oral and Maxillofacial Surgery, 45, 610617. https://doi.org/10.1016/j.ijom.2015.11.010

[2] Kafourou, V., Tong, H.J., Day, P., Houghton, N., Spencer, R.J. and Duggal, M. (2017) Outcomes and Prognostic Factors that Influence the Success of Tooth Autotransplantation in Children and Adolescents. Dental Traumatology, 33, 393-399. https://doi.org/10.1111/edt.12353

[3] Plotino, G., Abella Sans, F., Duggal, M.S., Grande, N.M., Krastl, G., Nagendrababu, V. and Gambarini, G. (2020) Clinical Procedures and Outcome of Surgical Extrusion, Intentional Replantation and Tooth Autotransplantation-A Narrative Review. International Endodontic Journal, 53, 1636-1652. https://doi.org/10.1111/iej.13396

[4] Park, J.H., Tai, K. and Hayashi, D. (2010) Tooth Autotransplantation as a Treatment Option: A Review. Journal of Clinical Pediatric Dentistry, 35, 129-135. https://doi.org/10.17796/jcpd.35.2.97816254u2140x88

[5] Gilijamse, M., Baart, J.A., Wolff, J., Sandor, G.K. and Forouzanfar, T. (2016) Tooth Autotransplantation in the Anterior Maxilla and Mandible: Retrospective Results in Young Patients. Oral Surgery, Oral Medicine, Oral Pathology, Oral Radiology and Endodontology, 122, e187-e192. https://doi.org/10.1016/j.oooo.2016.06.013

[6] Kumar, R., Khambete, N. and Priya, E. (2013) Successful Immediate Autotransplantation of Tooth with Incomplete Root Formation: Case Report. Oral Surgery, Oral Medicine, Oral Pathology and Oral Radiology, 115, e16-e21. https://doi.org/10.1016/j.00oo.2011.10.034 
[7] Staffoli, S., Plotino, G., Nunez Torrijos, B.G., et al. (2019) Regenerative Endodontic Procedures Using Contemporary Endodontic Materials. Materials (Basel), 12, E908. https://doi.org/10.3390/ma12060908

[8] Jang, Y., Choi, Y.J., Lee, S.J., Roh, B.D., Park, S.H. and Kim, E. (2016) Prognostic Factors for Clinical Outcomes in Autotransplantation of Teeth with Complete Root Formation: Survival Analysis for Up to 12 Years. Journal of Endodontics, 42, 198 205. https://doi.org/10.1016/j.joen.2015.10.021

[9] Higgins, J.P.T. and Green, S. (2011) Cochrane Handbook for Systematic Reviews of Interventions Version 5.1.0. The Cochrane Collaboration, Table 7.7.a: Formulae for Combining Groups.

[10] Patel, S. and Fanshawe, T. (2011) Survival and Success of Maxillary Canine Autotransplantation: A Retrospective Investigation. European Journal of Orthodontics, 33, 298-304. https://doi.org/10.1093/ejo/cjq071

[11] Grisar, K., Nys, M. and The, V. (2019) Long-Term Outcome of Autogenously Transplanted Maxillary Canines. Clinical and Experimental Dental Research, 5, 67-75. https://doi.org/10.1002/cre2.159

[12] Hewitt, A.B. and Gelson, W. (2011) Autotransplantation of Ectopic Teeth: A Retrospective Study of 284 Teeth. Oral Surgery, 4, 153-160.

https://doi.org/10.1111/j.1752-248X.2011.01129.x

[13] Grisar, K. and Smeets, M. (2020) Survival and Success of Auto Transplanted Impacted Maxillary Canines during Short-Term Follow Up: A Prospective Case-Control Study. Orthodontics \& Craniofacial Research, 24, 222-232. https://doi.org/10.1111/ocr.12422

[14] Huth, K.C. and Nazet, M. (2013) Autotransplantation and Surgical Up Righting of Impacted or Retained Teeth: A Retrospective Clinical Study and Evaluation of $\mathrm{Pa}$ tient Satisfaction. Acta Odontologica Scandinavica, 71, 1538-1546. https://doi.org/10.3109/00016357.2013.775667

[15] Andreasen, J.O., Paulsen, H.U., Yu, Z., Ahlquist, R., Bayer, T. and Schwartz, O. (1990) A Long-Term Study of 370 Autotransplanted Premolars. Part I. Surgical Procedures and Standardized Techniques for Monitoring Healing. European Journal of Orthodontics, 12, 3-13. https://doi.org/10.1093/ejo/12.1.3

[16] Grisar, K. and Chaabouni, D. (2018) Autogenous Transalveolar Transplantation of Maxillary Canines: A Systematic Review and Meta-Analysis. European Journal of Orthodontics, 33, 298-304.

[17] Verweij, J.P., Jongkees, F.A., Anssari Moin, D., Wismeijer, D. and van Merkesteyn, J.P.R. (2017) Autotransplantation of Teeth Using Computer-Aided Rapid Prototyping of a Three-Dimensional Replica of the Donor Tooth: A Systematic Literature Review. International Journal of Oral and Maxillofacial Surgery, 46, 1466-1474. https://doi.org/10.1016/j.ijom.2017.04.008

[18] Chung, W.C., Tu, Y.K., Lin, Y.H. and Lu, H.K. (2014) Outcomes of Autotransplanted Teeth with Complete Root Formation: A Systematic Review and MetaAnalysis. Journal of Clinical Periodontology, 41, 412-423.

https://doi.org/10.1111/jcpe.12228

[19] Atala-Acevedo, C., Abarca, J., Martínez-Zapata, M.J., Díaz, J., Olate, S. and Zaror, C. (2017) Success Rate of Autotransplantation of Teeth with an Open Apex: Systematic Review and Meta-Analysis. Journal of Oral and Maxillofacial Surgery, 75, 35-50. https://doi.org/10.1016/j.joms.2016.09.010

[20] Murtadha, L. and Kwok, J. (2017) Do Autotransplanted Teeth Require Elective Root Canal Therapy? A Long-Term Follow-Up Case Series. Journal of Oral and Maxillo- 
facial Surgery, 75, 1817-1826. https://doi.org/10.1016/j.joms.2017.03.049

[21] Lacerda-Santos, R. and Canutto, R.F. (2020) Effect of Orthodontic Treatment on Tooth Autotransplantation: Systematic Review of Controlled Clinical Trials. European Journal of Dentistry, 14, 467-482. https://doi.org/10.1055/s-0040-1708329

\section{Abbreviations}

PRISMA Preferred Reporting Items for Systematic Review and Meta-Analysis)

PROSPERO International Prospective Register of Systematic Reviews

PICOS Patient/Intervention/Comparison/Outcome study

MINORS Methodological Index for Non-Randomized Studies

MCAI Maxillary Canine Aesthetic Index

AMCRI Autotransplanted Maxillary Canine Radiological Index

RCTs Randomized Controlled Trials 